Printed in Great Britain

\title{
WALL DRAG ON FREE-MOVING CILIATED MICRO-ORGANISMS
}

\author{
BY H. WINET \\ Division of Engineering and Applied Science, California Institute of Technology, \\ Pasadena, California
}

(Received 22 May 1973)

\section{INTRODUCTION}

All aquatic micro-organisms whether swimming or merely sinking move in a bounded fluid. Nevertheless, motion studies of these bodies usually ignore any possible influence of the boundary or wall on their progress; and when acknowledged, wall effects are assumed to be insignificant. For example, in the classic study of Gray \& Hancock ( 1955 , p. 8I I) it is postulated that 'data on speeds of translation ... derived from [micro-organisms] . . . [swimming] in close proximity to a glass . . . surface ... also apply to [those] moving freely in a bulk of fluid'.

In a number of instances, however (e.g. sperm moving through narrow tubes, trypanosomes swimming in narrow blood vessels, immobilized ciliates sinking in glass tubes, organisms moving in thin slide preparations and oscillating bodies stuck to a wall) the wall is close enough to alter motion significantly. Indeed, if clearance between the cell surface and the wall is small enough, lubrication (i.e. flow like that around ball bearings) as well as non-hydrodynamic (e.g. electrostatic) effects may have to be taken into account.

A wall (or more specifically, the non-slip fluid layer against the wall) effects a nearby body's motion, of course, by encroaching upon its 'sphere of influence'; that is to say the volume of fluid reacting to the forces exerted by the body. That this reaction is essentially viscous stress is predicted by the low $R_{e}$ (Reynolds Number) characteristic of free-moving micro-organisms. Accordingly, one can refer to the volume in question as the organism's 'sphere of viscous influence, $(S)$ ' which is illustrated in Fig. I. The meanings of the symbols used in this paper are given in the Appendix.

Fig. I shows the essential differences between $S$ (of radius $S_{e}$ ) for sinking cells (of equivalent radius $R$ ) and $S$ (of radius $S_{w}$ ) for swimming cells (of minor semi-axis $a$ ). As is apparent from the diagram, $S_{w}<S_{e}$, because as indicated in the velocity profiles, a self-propelling body disturbs less of its surrounding fluid than a passive body does (see Blake, I973 and Jahn \& Votta, 1972, for more detailed velocity profiles of swimming bodies).

It is curious that even though wall effects are obviously significant at some clearance $\left(R_{0}-(R\right.$ or $a)$, or the form used here which is the non-dimensionalized 'reduced clearance' $R_{0} /(R$ or $a)-I$ ); and even though there must be a difference between $S_{e}$ and $S_{w}$, there are apparently no measurements of these quantities. Accordingly, it is the purpose of this work to obtain a measure of $S_{e}$ and $S_{w}$ by comparing the velocities $(U)$ of swimming and sinking ciliates down tubes of decreasing bore with their velocities 


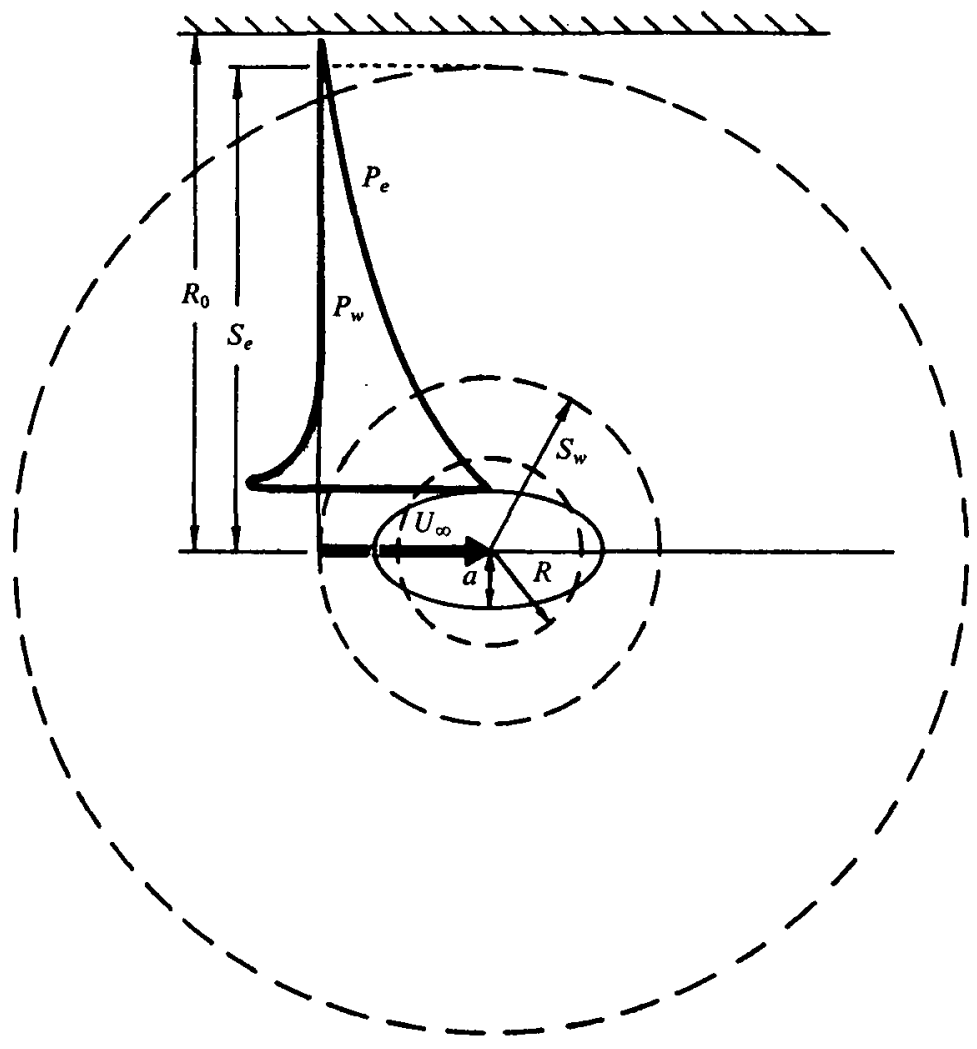

Fig. I. Velocity profiles of a self-propelled $\left(P_{w}\right)$ and a passively moving $\left(P_{e}\right)$ body at low $R_{a *}$ A body of minor semi-axis a moves its centroid from the base to the tip of the horizontal arrow at a constant velocity $U_{\infty}$. If passive, the body entrains fluid by an outwardly decreasing amount indicated by velocity profile $P_{\bullet}$. If oscillating, it entrains very little fluid as indicated by profile $\boldsymbol{P}_{w}$. Theoretically, $\boldsymbol{P}_{a}$ becomes asymptotic to the vertical axis while $\boldsymbol{P}_{w}$ merges with it at a distance $S_{w}$ from the translational axis. An experimentally practical cutoff or pseudomerging point is determined at $S_{0}$ from the translational axis. $S_{0}$ and $S_{w}$ are, then, radii of the spheres of viscous infiuence of passive and propulsive movers, respectively. Note, however, that the $S_{o}$ and $S_{w}$ referred to in the text are the radii of the spheres of viscous influence minus one equivalent cell radius $(R)$ or minor semi-axis $(a)$; i.e. they are clearances.

The solid boundary at $R_{0}$ from the translational axis has no significant effect on $U_{\infty}$ until $R_{0}<S_{b}$ or $R_{0}<S_{w}$ for a sinking or swimming cell, respectively. The equivalent sphere (of radius $R$ ) can represent the passive body with no effect on the data until $R_{0} \leqslant S_{6}$.

in an unbounded fluid $\left(U_{\infty}\right)$ and with each other. After determining $S_{e}$ and $S_{v}$, their values will be compared with corresponding theoretical models which estimate spheres of viscous influence from the point of view of the validity of Stokes equations for motion of a body at low $R_{e}$.

\section{MATERIALS AND METHODS}

Paramecium caudatum and P. multimicronucleatum grown in wheat-fortified Paramecium medium (Ward's) and Tetrahymena sp. (from Ward's) grown in $2 \%$ proteosepeptone were observed in glass tubes at $20^{\circ} \mathrm{C}$. In all cases the portion of the tube being viewed was immersed in immersion oil to reduce refraction and to enable measurement to be made of the tube bore. 


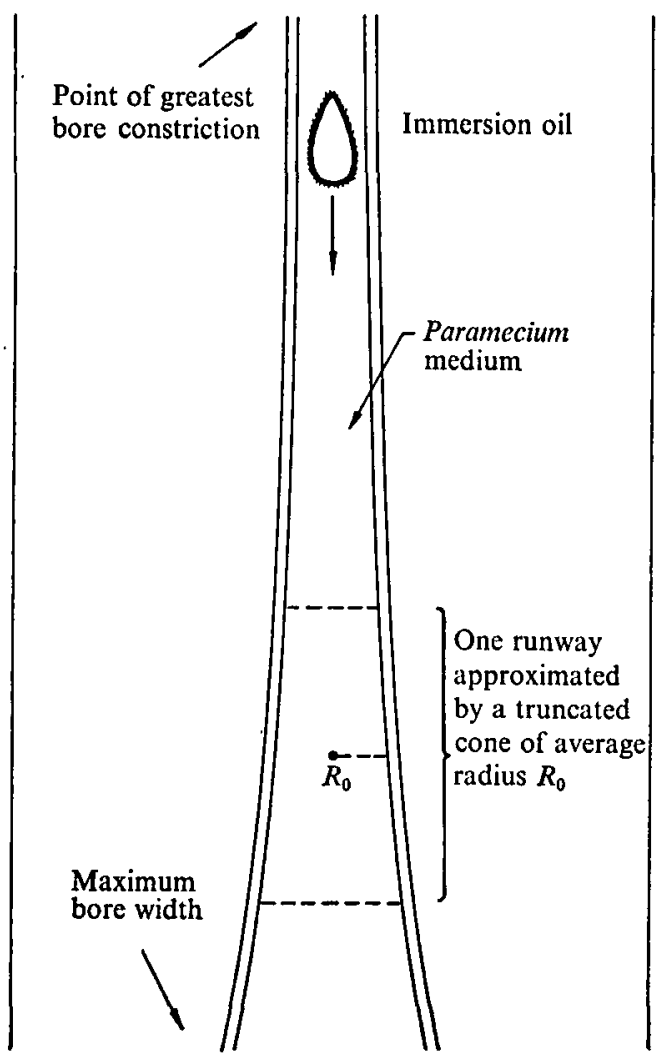

Fig. 2. A sedimentation tube. The tube is immersed in immersion oil to allow resolution of the inner bore which varies continuously from the point of constriction to the original tube width. Sinking paths were less than I mm, so the fluid volume containing the cells could be approximated by a truncated cone. The average diameter of the cone is, then, the bore width $\left(2 R_{0}\right.$ in figure).

\section{(A) Sedimentation}

Ciliates to be sedimented were fixed in formaldehyde and rinsed in Paramecium medium at least 3 times by hand centrifugation, decantation and re-suspension. Sedimentation in vertical (determined by eye with a plumb line) constricted tubes was followed with a horizontal binocular dissecting microscope fitted with a calibrated Whipple disc and timed with a hand-held stopwatch. Only cells at the point of narrowest bore or below and those tilted $\sim 45^{\circ}$ or less from the vertical axis were timed. In the swimming experiments cells were timed over a course of constant bore; in the sinking experiments the bore width of the vertical tubes increased to a maximum below the constriction. Accordingly, only the average width for each course length was utilized. An example of the tube structure is presented in Fig. 2. Sample sizes ranged from 13 upwards with one exception: only 2 measurements could be obtained for $P$. multimicronucleatum at the narrowest tube bore.

The physical sedimentation model consisted of $2 \mathrm{~mm}$ plastic spheres sinking in a series of vertical glass tubes of different bores which were filled with Dow Corning DC 200 fluid (rooo centistokes). Sinking velocity was determined from photographs of ftroboscopic flashes taken during the descent of the sphere. 
In order to determine $S_{e}$ in a bounded fluid the measured velocities $U$ had to compared with the corresponding velocity $U_{\infty}$ in an unbounded fluid. The latter exists, of course, only hypothetically. Accordingly, $U_{\infty}$ had to be determined theoretically, and three methods were utilized because each could claim validity, given suitable data.

(I) The fastest measured $U$ may be taken as the maximum $U$ value. Thus, if this $U$ is given the symbol $U_{\infty \text { meas }}$ we have

$$
U_{\infty \text { meas }}=U_{\infty} \text {. }
$$

(2) If cell density is known the sedimentation equation may be utilized.

$$
U_{\infty}=U_{\infty \text { est }}=\frac{2 r^{2} g\left(\rho-\rho_{0}\right)}{9 \eta},
$$

where the sphere radius $r$ may be replaced for a non-spherical body by the equivalent radius $R$ under low $R_{e}$ conditions (see Happel \& Brenner, 1965, p. 223) and $\rho$ for the ciliates is assumed to be that of Tetrahymena pyriformis (calculated as $\mathrm{I} \cdot 076$ from Dunham \& Child, r96r).

3. An interpolated $U_{\text {clnt }}=U_{\infty}$ may be calculated from the ratio of Stokes drag on a spheroid in an unbounded fluid $\left(F_{s \infty}\right)$ to the numerical solution for Stokes drag on a spheroid settling in a cylinder with the major axis of the spheroid collinear with the axis of the cylinder $\left(F_{s b}\right)$ (Happel \& Brenner, I965, p. 340).

$$
\left.U_{\text {cint }}=\frac{F_{s b}}{F_{s \infty}} U=\frac{U}{\mathrm{I}-\mathrm{I} \cdot 43\left(c / R_{0}\right)\left[5 \cdot 6 \mathrm{I} 2-2 \cdot 02 \mathrm{II}\left(a / R_{0}\right)^{2}-3 \cdot 543 \mathrm{I}\left(c / R_{0}\right)^{2}\right.}\right] .
$$

Here $R_{0}$ is the internal radius of the tube, $a$ is the minor semi-axis of the spheroid and $c$ is its major semi-axis. A more exact formula is available for the sphere model

$$
\frac{U}{U_{\infty}}=\frac{F_{s \infty}}{F_{s b}}=\frac{\mathrm{I}-2 \cdot 1050\left(b / R_{0}\right)+2 \cdot 0865\left(b / R_{0}\right)^{5}+0.72603\left(b / R_{0}\right)^{6}}{\mathrm{x}-0.75^{8} 57\left(b / R_{0}\right)^{5}},
$$

(Happel \& Brenner, I965, pp. $3^{\mathrm{r} 8-20}$ ) where $U_{\infty}=U_{\infty \text { est }}$ which is readily calculated from the measured density of the plastic sphere and the known kinematic viscosity of DC 200.

To avoid geometric effects due to the asymmetry of a ciliate the values of $U$ chosen for calculating $U_{\text {oint }}$ were limited to those measured at maximum $R_{0}$.

Finally, the determination of the radius of $S_{e}$ as the distance for significant wall drag followed a study of the plots of $U / U_{\infty}$ as a function of $R_{0}$, the details of which are reserved for the conclusion section.

\section{(B) Srvimming}

Swimming cells were timed with a hand-held stopwatch as they swam over a premeasured distance in a horizontal capillary tube. The tubes of narrower bore were obtained by drawing out the tubing over a flame. For trials with large-bore tubes, only those cells having swimming paths of small amplitude were measured (ciliates swim along helical paths, i.e. they gyrate). The dimensions of the cell and of the tube bore were determined from photomicrographs or measured directly through the microscope with the aid of a calibrated Whipple disk. Sample sizes ranged from 20 upwards. 


\section{Table I. Sinking velocity ratios}

(All symbols are defined in the text. $U_{\infty \text { est }}$ is calculated assuming a viscosity of $10^{-2} \mathrm{~g} / \mathrm{cm}$ sec for the ciliates. The density of the plastic spheres is $I \cdot 176$. Only measured velocities are included in the $R_{s}$ range. The starred (*) value was obtained from Kuznicki, I968.)

\begin{tabular}{|c|c|c|c|c|}
\hline & Tetrahymena & P. caudatum & P. multimicronucleatum & $2 \mathrm{~mm}$ sphere \\
\hline$a\left(\times 10^{4} \mathrm{~cm}\right)$ & $8 \cdot 35 \pm r \cdot 5$ & $39.5 \pm 6.0$ & $45 \cdot 5 \pm 5 \cdot 5$ & 1000 \\
\hline$c\left(\times 10^{4} \mathrm{~cm}\right)$ & $13.00 \pm 3.25$ & $89 \cdot 0 \pm 7.5$ & $113.5 \pm 17.5$ & 1000 \\
\hline$R\left(\times 10^{4} \mathrm{~cm}\right)$ & $9 \cdot 0$ & $50 \cdot 0$ & $60 \cdot 0$ & 1000 \\
\hline$R_{0} ; U\left(\times 10^{4} \mathrm{~cm}\right)$ & $\begin{array}{l}125 ; 4.45 \pm I \cdot 39 \\
650 ; 8.55 \pm 3 \cdot 83\end{array}$ & $\begin{array}{rr}250 ; & 27.8 \pm 7.6 \\
650 ; & 46.4 \pm 15.6 \\
900 ; & 61.5 \pm 17.3 \\
2000 ; & 69.8 \pm 20.7 \\
3050 ; & 78.6 \pm 15.7 \\
6700 ; & 107.0 \pm 23.0\end{array}$ & $\begin{aligned} & 95 ; 22 \cdot 3 \\
& 205 ; 39.0 \pm 7.4 \\
& 550 ; 58.2 \pm 14.2 \\
& 1650 ; 65.5 \pm 10.5 \\
& 2400 ; 68.6 \pm 15.2 \\
& 6700 ; 145.0 \pm 23.0\end{aligned}$ & $\begin{array}{r}2400 ; 118 \pm 2.0 \\
3000 ; 161 \pm 5.5 \\
4600 ; 232 \pm 7.0 \\
6850 ; 299 \pm 3.0 \\
26050 ; 389 \pm 12.0\end{array}$ \\
\hline$R_{0}$ range & $\begin{array}{l}8 \times 10^{-5}- \\
1.54 \times 10^{-4}\end{array}$ & $\begin{array}{l}2.78 \times 10^{-3}- \\
1.07 \times 10^{-8}\end{array}$ & $\begin{array}{r}2.64 \times 10^{-3}- \\
1.74 \times 10^{-2}\end{array}$ & $\begin{array}{l}2.29 \times 10^{-4}- \\
7.55 \times 10^{-4}\end{array}$ \\
\hline$U_{\infty \text { est }}(\mathrm{cm} / \mathrm{sec})$ & $1 \cdot 53 \times 10^{-3}$ & $3.79 \times 10^{-2}$ & $5.45 \times 10^{-2}$ & $4.33 \times 10^{-2}$ \\
\hline$U_{\infty \operatorname{lnt}}(\mathrm{cm} / \mathrm{sec})$ & $9.7 \times 10^{-4}$ & $I \cdot 14 \times 10^{-2}$ & $1.56 \times 10^{-8}$ & $4.37 \times 10^{-2}$ \\
\hline
\end{tabular}

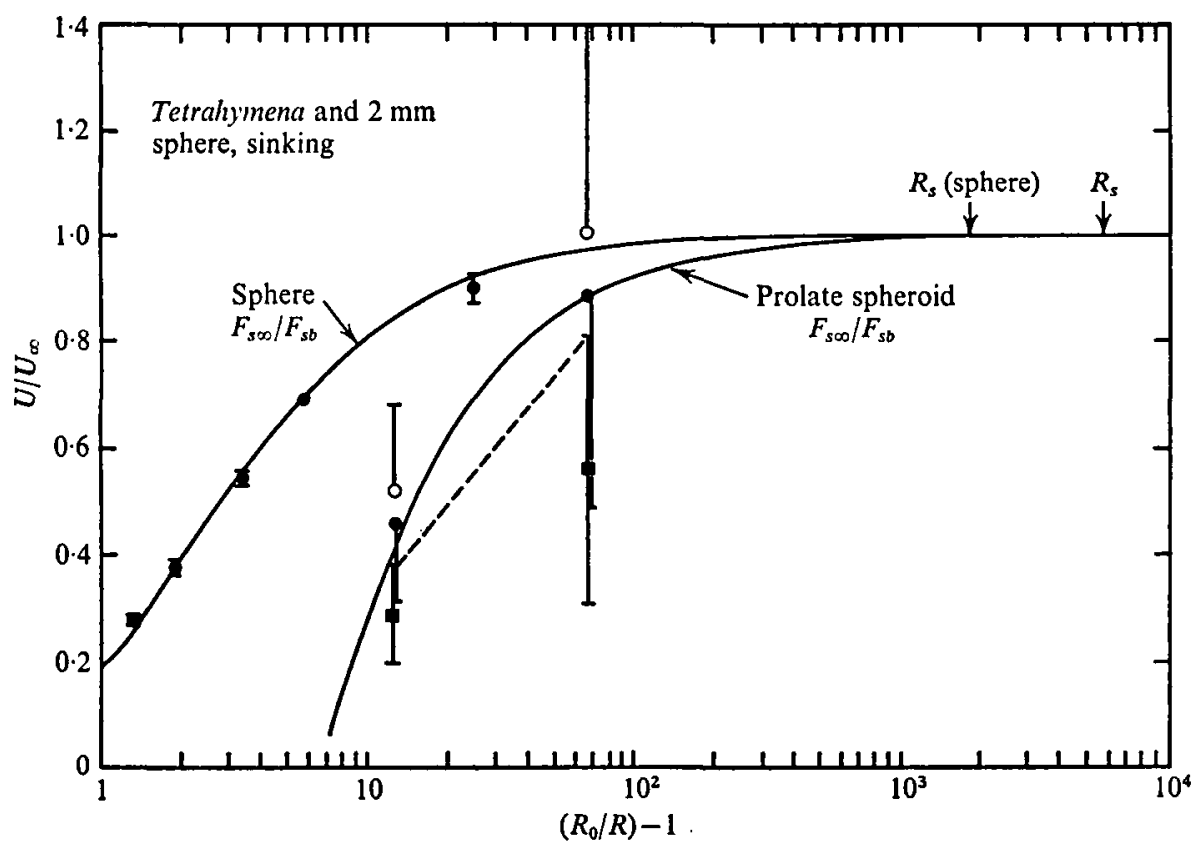

Fig. 3. Velocity ratio of sinking Tetrahymena sp. and plastic spheres as a function of reduced clearance. The two solid lines represent plots of equation (3) and (4) which represent the spheroid approximating the ciliate body and the plastic sphere respectively. Variances are standard deviations of the sample. In the figure the open circles represent plots of $U / U_{\infty}$ where $U_{\infty}$ is defined in ( 1 ), the closed circles represent plots of (3) or (4) and the squares represent plots of (2). All symbols except $R_{8}$ are defined in Materials and Methods. The Stokes radius $R_{s}$ is defined in the Discussion. Of the three forms of $U / U_{\infty}$ it will be noted that $U / U_{\infty \text { est }}$ underestimates $F_{s \infty} / F_{s b}$ for this ciliate while showing good agreement for the physical model. The other two forms of $U / U_{\infty}$ which were used for the ciliates only show good agreement with the mathematical model (3). 


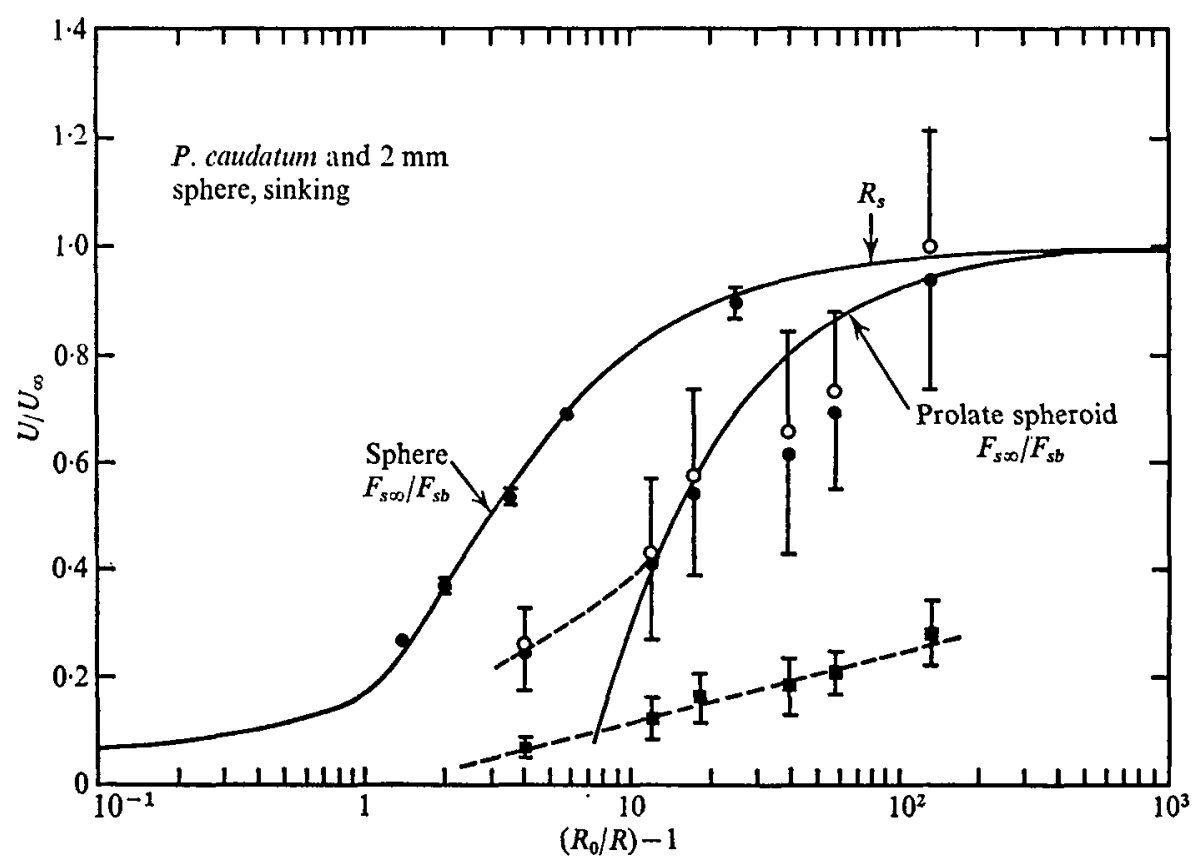

Fig. 4. Velocity ratio of sinking $P$. caudatum and plastic spheres as a function of reduced clearance. All plots and symbols have the same meaning as in Fig. 2. Here the underestimation of $F_{s \infty} / F_{s b}$ by ciliate $U / U_{\infty \text { est }}$ is more marked than the case for Tetrahymena. In addition a tendency for both $U / U_{\infty \text { int }}$ and $U / U_{\infty \text { mess }}$ to underestimate the drag ratio is detectable between $U / U_{\infty}=0.54$ and 0.92 . Deviation of the model (3) from data at reduced clearance values below ro are due to the breakdown of the model (which is, after all, a polynomial numerical solution) close to the moving surface.

\section{RESULTS}

\section{(A) Sedimentation}

Data from sinking cells were collected until $R_{0}$ reached the practical limits of focal length and optical resolution. Measurements are summarized in Table I. As expected from the drag-ratio models $(3,4), U$ increases with $R_{0}$. Similarly, $R_{e}$ increases with $R_{0}$ by an order of magnitude in the case of Paramecium.

While $U_{\infty \text { est }}$ and $U_{\infty \text { int }}$ are almost identical for the physical model, as expected, there is significant discrepancy between the two measures for all the ciliates. In contrast, the discrepancies between $U_{\infty \text { meas }}$ and $U_{\infty \text { int }}$ fall well within the standard deviation of the former for all three ciliates.

These relationships are more striking when sinking velocity ratio is plotted as a function of reduced clearance, as shown in Figs. 3-5. In all three graphs the solid lines represent the mathematical models utilizing the measured values of $R$. The agreement of the physical model with (4) is quite good. The spheroid mathematical model (3) does, however, tend to overestimate sinking velocity for all three ciliates regardless of the method for calculating $U_{\infty}$. Except in the case of $P$. multimicronucleatum, this tendency does remain within the standard deviations of $U / U_{\infty \text { meas }}$ for clearance values beyond Io $\left(R_{0} / R-I\right)$ (namely, ro cell equivalent radii) and deviates less than $3 \%$

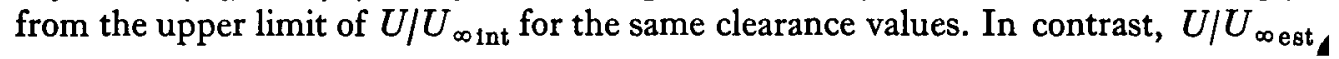




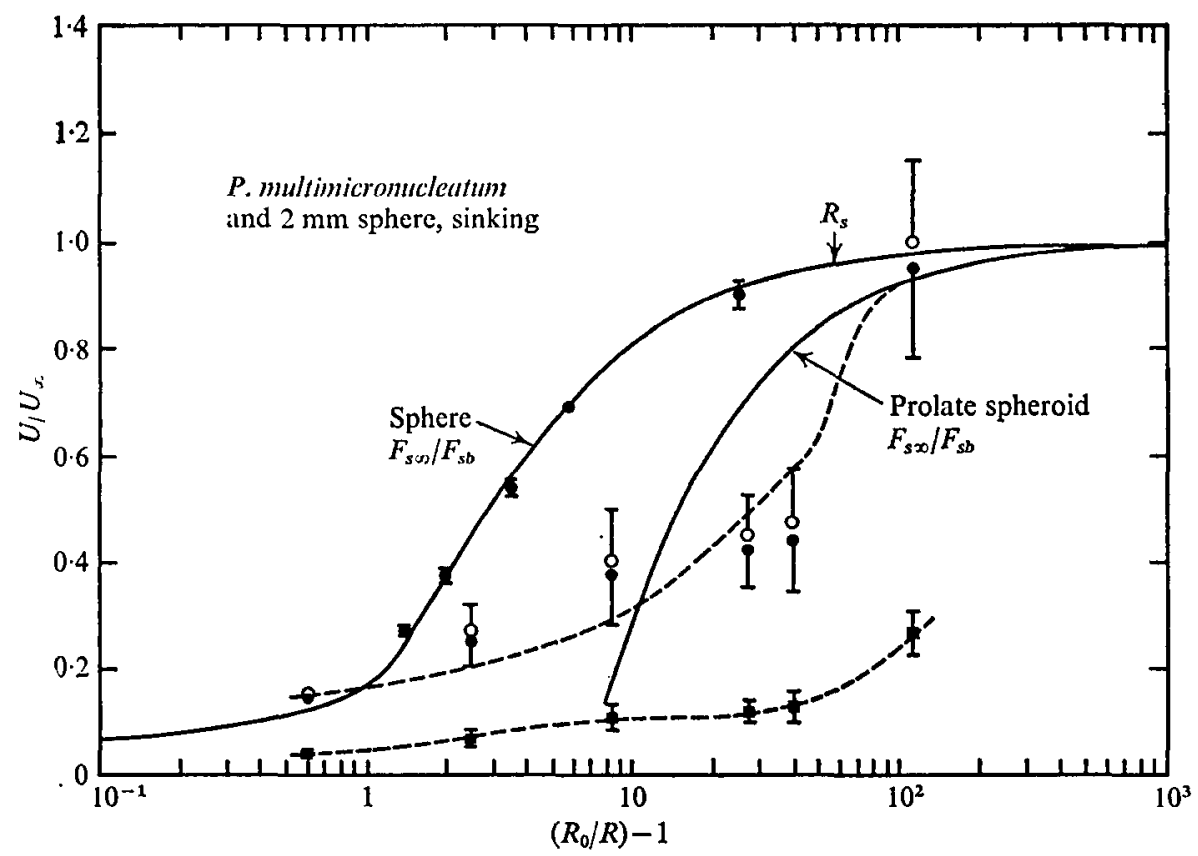

Fig. 5. Velocity ratio of sinking $P$. multimicronucleatum and plastic spheres as a function of reduced clearance. All plots here are similar to those for $P$. caudatum only more deviant. Only one value of $U / U_{\infty \text { int }}$ and one of $U / U_{\infty \text { meas }}$ fall on the $F_{s \infty} / F_{s b}$ plot. Overestimation by the model between velocity-ratio values of 0.35 and 0.92 reaches $22 \%$ at one point. Nevertheless, the agreement of $U / U_{\infty \text { mens }}$ with the model at the maximum measured value does establish an upper limit for the clearance at which there is 'safety' from wall drag.

Table 2. Sample spheroid velocity ratios for given reduced clearances

All symbols are defined in the text.

$\begin{array}{cc}\text { Reduced } \\ U / U_{\infty \text { Int }} & \begin{array}{c}\text { clearance } \\ \left(R_{0} / R\right)-I\end{array} \\ 0.50 & 15.1 \\ 0.60 & 19.3 \\ 0.80 & 38.5 \\ 0.90 & 80.0 \\ 0.95 & 166.7\end{array}$

approaches that for the model only in the case of Tetrahymena, being the lowest curve in all three cases.

The plot of (3) is virtually identical in Figs. 3-5. Accordingly, one set of velocityratio values and corresponding clearances obtained using the model may be used with all three ciliates. Such a set is presented in Table 2.

\section{(B) Swimming}

Data from swimming ciliates are summarized in Table 3 . A comparison with Table I shows a maximum velocity ratio between swimming and sinking cells $\left(U_{\infty \mathrm{sw1m}} / U_{\infty \text { int }}\right)$ ranging from 7.5 to 50 with decrease in $R$ or $a$. Unlike sinking cells, the swimmers 


\section{Table 3. Swimming velocity ratios}

( $U_{\infty \text { swim }}$ is the maximum swimming velocity. All other symbols are defined in the text.)

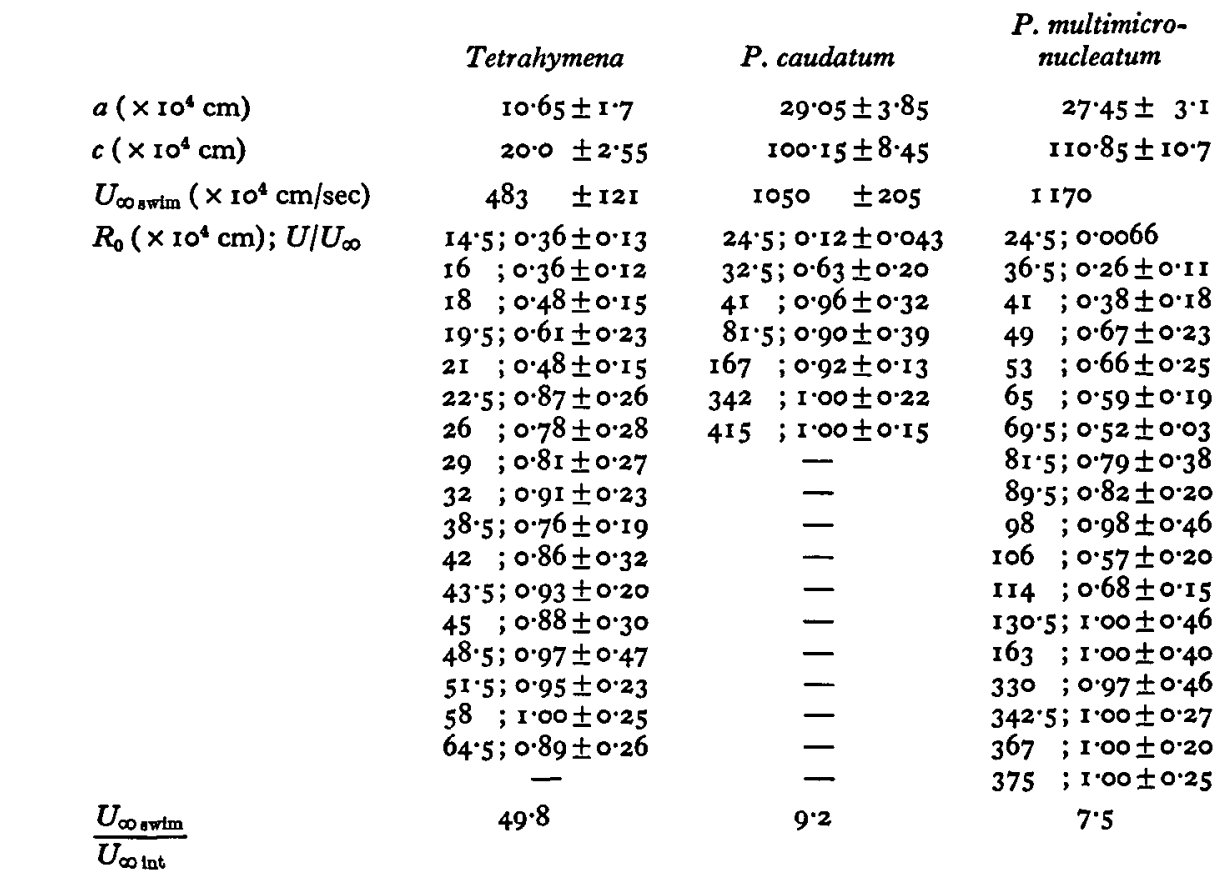

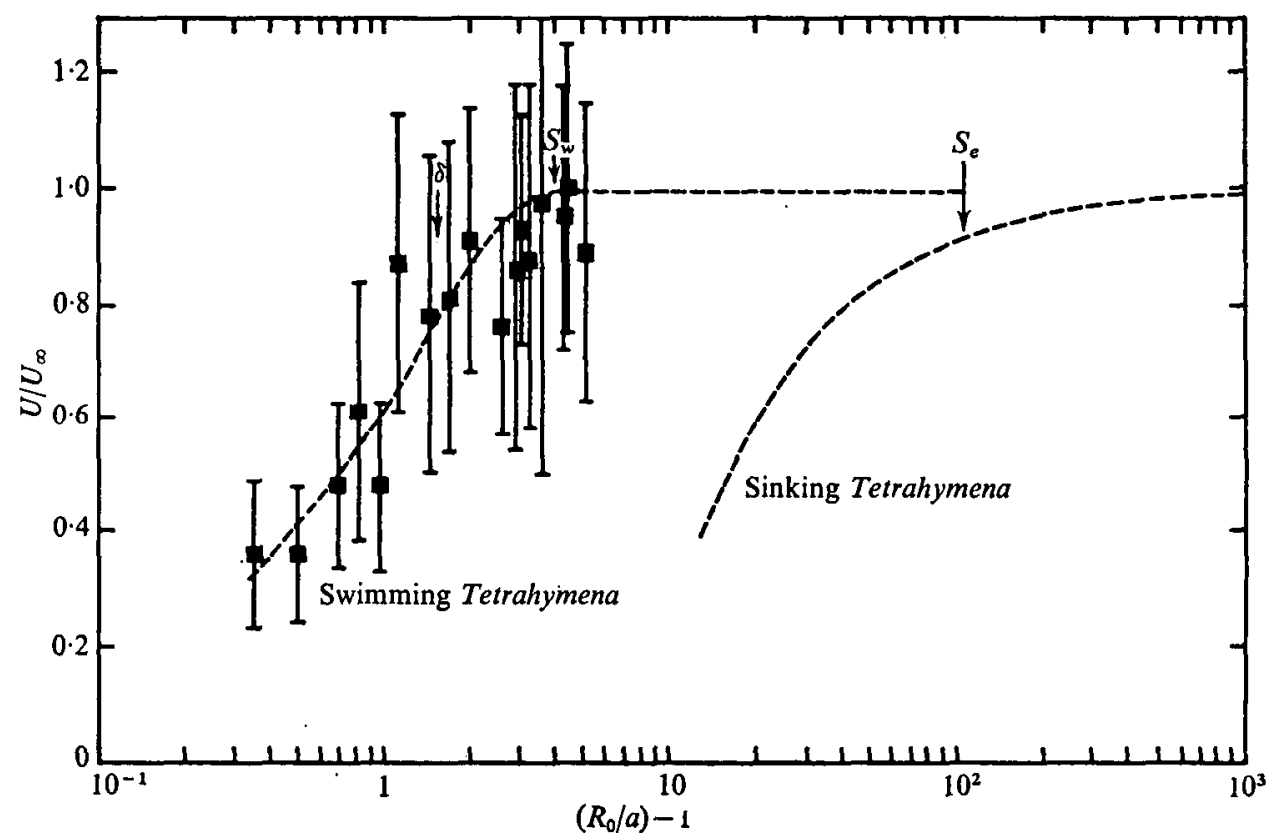

Fig. 6. Velocity ratio of swimming Tetrahymena $\mathrm{sp}$. as a function of reduced clearance is traced by the heavy dashed line. Velocity in an 'unbounded' fluid $U_{\infty}$ swm is reached at a clearance of $S_{w}$. The light dashed line represents corresponding data from sinking Tetrahymena which reaches an effective maximum velocity at a clearance of $S_{c}$. The symbol $\delta$ represents the radius of the region of validity of the quasi-steady Stokes equation or the depth of penetration of the vorticity which is dealt with in the Discussion. All variances are standard deviations of the sample. 


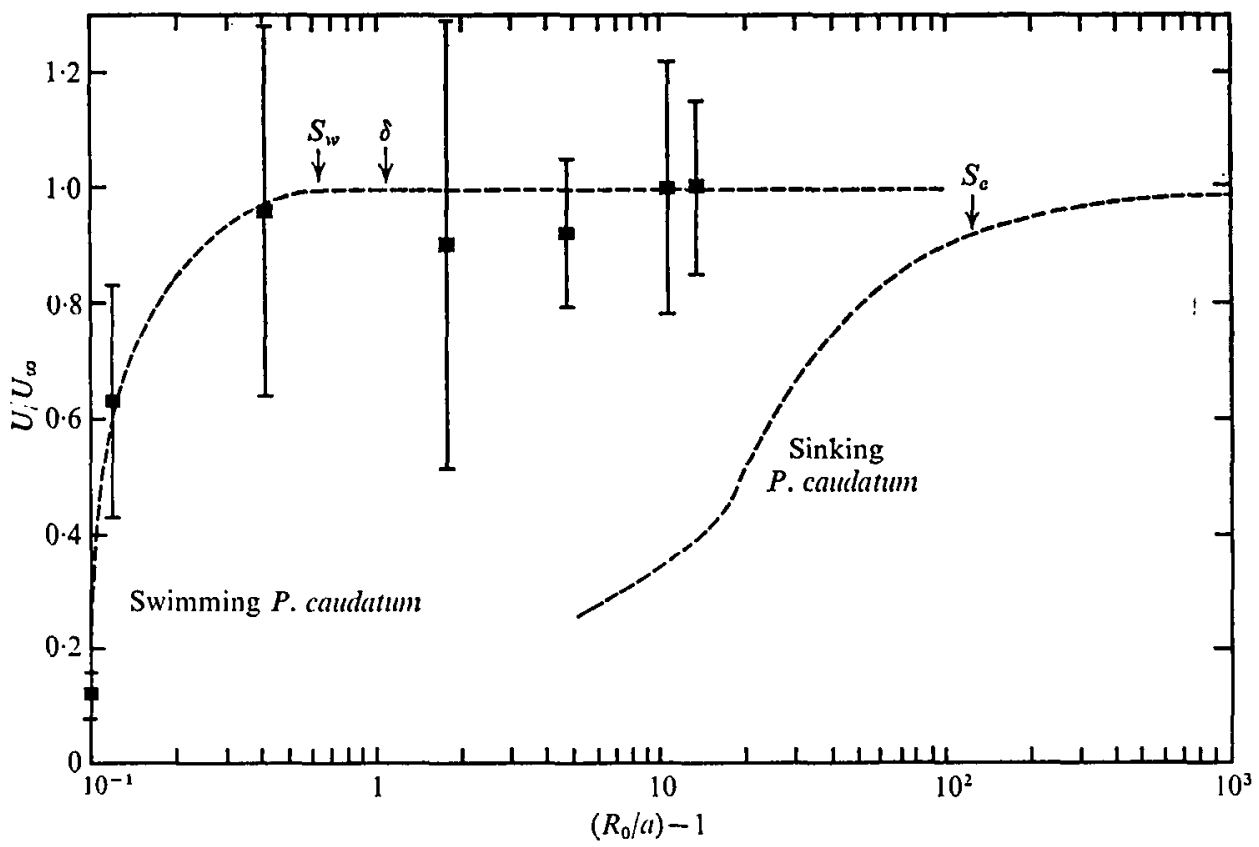

Fig. 7. Velocity ratio of swimming $P$. caudatum as a function of reduced clearance. The pattern of this figure is the same as that for Fig. 5.

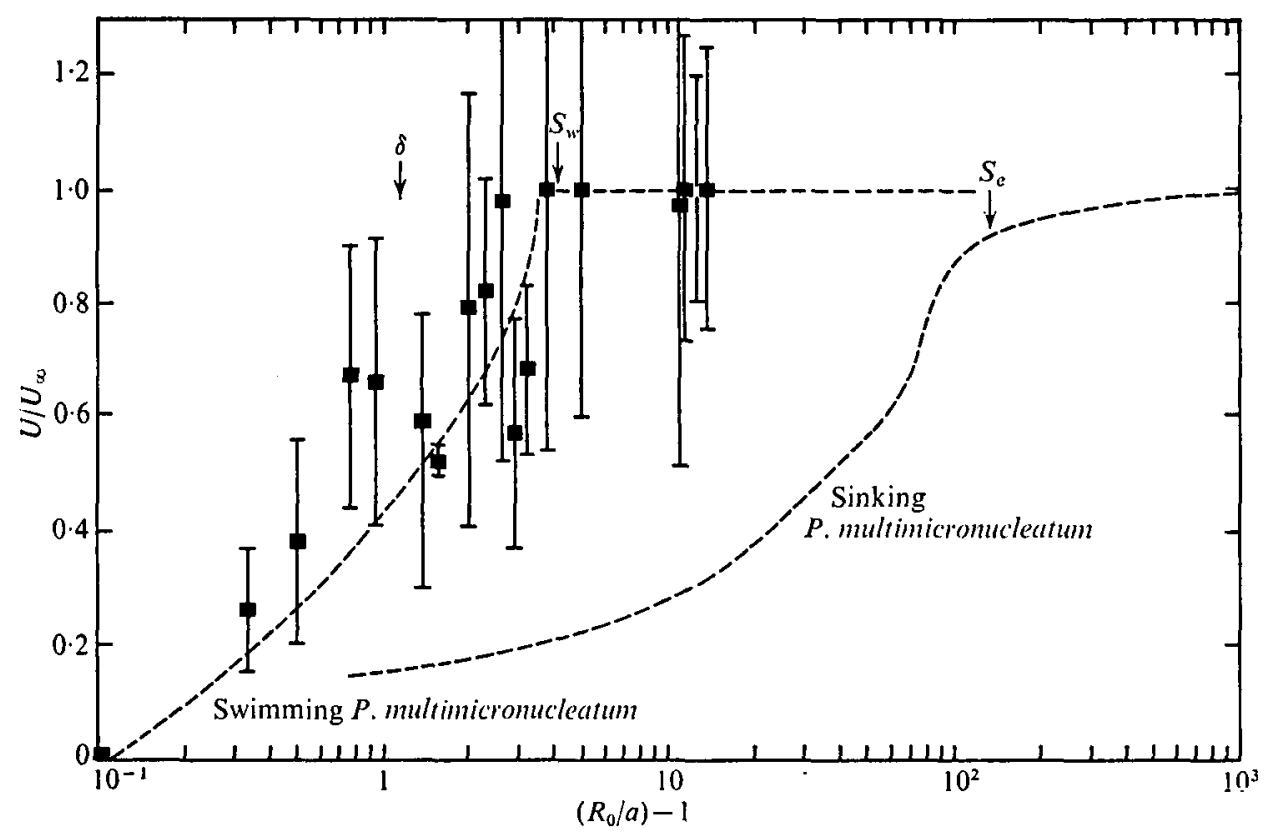

Fig. 8. Velocity ratio of swimming $P$. multimicronucleatum as a function of reduced clearance. The pattern of this figure is the same as that for Fig. 5. 
Table 4. Comparison of velocity profiles or radii spheres of viscous influence $(S)$ for sinking and for swimming ciliates

(The passive/propulsive ratio $\left(S_{e} / S_{w}\right)$ given for $P$. caudatum may be larger than its real value (cf. Fig. 6 which has relatively few points when compared with Figs. 5, 7).)

$\begin{array}{lccc} & \text { Tetrahymena } & \text { P. caudatum } & \begin{array}{c}\text { P. multi- } \\ \text { micronucleatum }\end{array} \\ S_{8} & 108 a & 127 a & 132 a \\ S_{w} & 4 \cdot 1 a & 0.64 a & 4.2 a \\ S_{s} / S_{w} & 27 & 198 & 31 \cdot 4\end{array}$

achieve a maximum $U$ at $R_{0}$ values well within the focal length and optical resolution of the observation system. Accordingly, $U_{\infty}$ was measured directly, enabling $U / U_{\infty}$ to be plotted independently of any model and simply as a function of clearance. The resultant graphs are presented in Figs. 6-8. Here it is apparent that $U / U_{\infty}=\mathrm{I}$ is maintained to within the following distances of the ciliate body surface: Tetrahymena, $4 \cdot \mathrm{r} a ; P$. caudatum, $0.64 a ; P$. multimicronucleatum, $4 \cdot 2 a$. A plot of the sedimentation data is included in the figures for comparison.

\section{CONCLUSIONS}

Equation (3) may be considered adequate for determining the value of $S$ safe from wall drag on the body of a sinking ciliate. If there is error in the estimate it is in the desirable direction of overrating, with a maximum error of about $22 \%$ (see Fig. 5). The difference between the plots for the sphere model and for the prolate spheroid model, together with the dependence of both (3) and (4) on sphericity and axisymmetry, leads to the conclusion that this error is a function of geometry. That is to say, given the shape of Tetrahymena, essentially an intersection of a paraboloid and ellipsoid of revolution (Winet, I969), and the shape of Paramecium, being non-axisymmetric because of the large oral groove, one should not expect to obtain velocity measurements in agreement with those obtained using a model based upon viscous stresses over a spheroid. However, the measure of $S_{e}$ as the distance at which wall drag is significant is the concern here, and the errors described above are important only to the extent that they determine $S_{e}$ by indicating the point at which the wall can 'discriminate' departures of the sinking bodies from the spheroid shape. The clearest indication of such a point appears in Fig. 5 where the measured values for $P$. multimicronucleatum deviate markedly from those for the model and indicate that shape 'discrimination' can occur up to about $U / U_{\infty}=0.92$. This velocity ratio corresponds to a reduced clearance of $100 R$.

Accordingly, $S_{e}=100 R$ which in terms of $a$ yields the following: Tetrahymena, 108a; $P$. caudatum, 127a; $P$. multimicronucleatum, 132a.

In contrast, when these ciliates are swimming the wall cannot 'discriminate' them until it is within $\sim 4 a$ of their body surfaces. Such values of $S_{w}$ when compared with $S_{e}$ are consistent with the qualitative relationship between the velocity profiles described in Fig. I. They are also summarized in Table 4 in the form of a passive/propulsive ratio from which it is evident that the height of $S_{e}$ for these ciliates is 27-r98 times as high as that of $S_{w}$. The specific profiles for each ciliate are approximated by their 
locity-ratio graphs as presented in Figs. 6-8. The comparison may best be made by rotating the figures $90^{\circ}$ counter-clockwise.

The most obvious conclusion to be drawn from the $U / U_{\infty \text { est }}$ data is that formalinfixed specimens of the tested ciliates do not have the same value of $\rho$ as $T$.pyriformis. Either these organisms are inherently less dense than their model or they were rendered so by the formalin. Although both mechanisms may be valid, the similarity of the Paramecium plots and their divergence from the Tetrahymena plot point to an inherent density difference.

\section{DISCUSSION}

The values of $S_{e}$ and $S_{w}$ obtained above are useful as 'boundary conditions' for experimental work with moving micro-organisms. But the question arises as to their utility for the hydromechanical models being applied to these experiments. This question is most pertinent because the present model, the Stokes equations, is valid only within a certain distance of the moving body, and data on fluid flow outside this distance will not conform to the model. Hence, there is just as much need to establish a 'safe' sphere of 'influence' for the theoretical model as there is for the experimental objects. The analogy between validity limits on theoretical and experimental results is worth stressing as it makes the former seem more 'real' and, hence, easier to accept as a model for observations. Actually, solutions to Stokes equations in their quasisteady form (for oscillating bodies translating at steady velocities) for non-rotating waving bodies (Gray \& Hancock, 1955) and for rotating waving bodies (Chwang \& Wu, I97I) have already generated formulas which agree satisfactorily with observations (Gray \& Hancock, 1955; Gray, 1962; Chwang, Wu \& Winet, 1972; Chwang, Winet \& Wu, I973).

Hence, just as it is necessary for the purpose of gathering data to know when the assumption of 'no wall effect' is invalidated, it is similarly important for the purpose of matching data with an approximate model to know where the two are expected to diverge; e.g. it will be noted that (3) diverges from the data at reduced clearance values below ro (see Figs. 4, 5). This result is expected because (3) is a polynomial approximation which begins to oscillate at low values of the independent variable. For a body in steady motion (a sinking cell at terminal velocity) the critical distance is termed the Stokes radius $\left(R_{s}\right)$ and the corresponding distance for a body in quasi-steady motion is the 'depth of penetration of the vorticity' $(\delta)$. Both $R_{s}$ and $\delta$ are mathematical quantities not intended for expression in physical terms. Nevertheless, some physical concept of these quantities is necessary for the comparison to be made below; and, accordingly, they may be represented by the models shown in Figs. 9, Io. In addition, an estimate of these critical distances may be calculated from

$$
R_{s}=R / R_{e} \text { or } \mathrm{I} / R_{e} \text { (in terms of reduced clearance) }
$$

(Rosenhead, 1963) where $R_{e}=U_{\infty} R / \nu, \nu$ is the kinematic viscosity,

$$
\delta=\frac{\lambda}{\left(\mathrm{I}+R_{\Theta \omega}^{2}\right)^{\frac{1}{t}}}(\mathrm{~J} . \text { Blake, personal communication })
$$

$R_{e \omega}=\omega / \nu k^{2}$ is the oscillatory Reynolds Number, $\omega$ is the frequency of ciliary beat (obtained from Machemer, 1972 , and Preston, 1972 ), $k=2 \pi / \lambda$, and $\lambda$ is the metachronal ravelength (obtained from Machemer, 1972, and Parducz, 1966). 


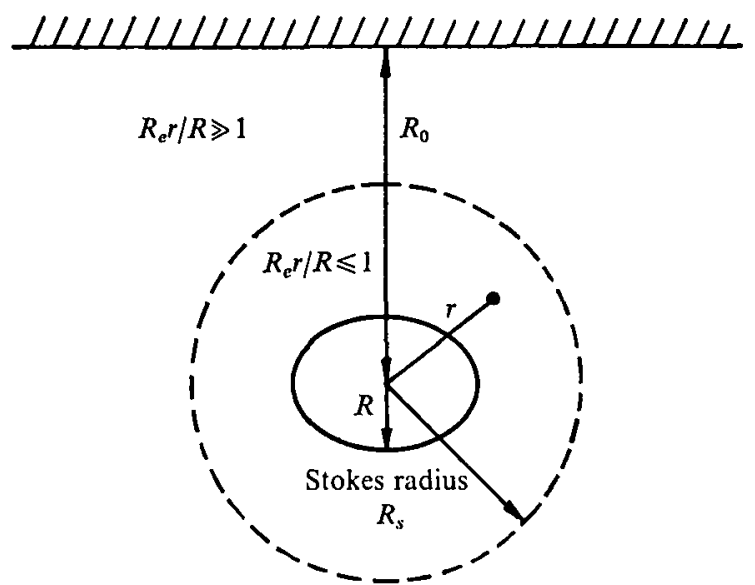

Fig. 9

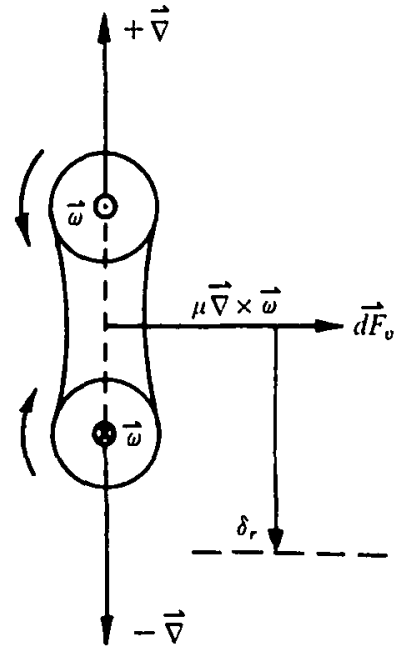

Fig. 10

Fig. 9. A physical conception of the Stokes radius $\left(R_{s}\right)$. Stokes equations for steady motion of a body are valid out to the point where $r=R_{g}$. Beyond this point inertial and convective stresses are so large in comparison with diffusion and viscous stresses that the Oseen correction must be introduced to the Stokes equations making them much more difficult to solve. The question taken up in the Discussion is: 'Is $S_{a}$ of the same order as $R_{s}$ ?'.

Fig. Io. A physical conception of the depth of penetration of the vorticity $(\delta)$. Stokes equations for quasi-steady motion of a body are valid out to the distance $\delta_{r}$ which is greater than $\delta$ by one minor semi-axis of the cell. It has been shown (see Taylor, 1952) that micro-organism propulsion is generated by a viscous-stress-induced rotation of fluid elements surrounding an oscillating body. In effect, a torus of fluid develops around each infinitesimal body segment which rotates about its material axis such that the torus surface in contact with the body thrusts it forward in the direction opposite $d \vec{F}$. This thrust can be shown mathematically to be due entirely to the cross product of the vorticity $\vec{\omega}$ or rotation tendency (actually equal to twice the angular velocity of the fluid elements on the axis just described) and the gradient $\vec{\nabla}$ along which such vorticity diffuses outward from the body. Beyond $\delta$, as in the case for $R_{\mathrm{g}}$, the uncorrected Stokes equations are not valid. The question taken up in the Discussion is: 'Is $S_{w}$ of the same order as $\delta$ ?'.

It will be noted that both $R_{s}$ and $\delta$ are functions of $R_{e}$ while (3) and (4) within the confines of a 'low $R_{e}$ ', are not. Yet, when wall drag slows a cell it must be altering body $R_{e}$ (see Table $\mathrm{I}$ ). Consequently, $R_{s}$ and $\delta$ may be considered as functions of wall effect (i.e. clearance) through resultant changes in body $R_{e}$. Furthermore, since it is easier to perform experiments like those reported here than to obtain some of the parameters in (5) and (6), it is a clear advantage to be able to estimate $R_{s}$ with $S_{e}$ and $\delta$ with $S_{w}$. In effect, such a technique would match the models in Figs. 9, 10 with those in Fig. $\mathrm{I}$.

Toward this end, calculated values for $R_{s}$ are plotted in Figs. 3-5, those for $\delta$ are plotted in Figs. 6-8, and a comparison of all four quantities is listed in Table 5 .

Examination of corresponding values of $R_{s}$ and $S_{e}$ reveals a poor match for Tetrahymena but a not unreasonable fit for Paramecium, given the asymptotic nature of th 
able 5. Comparison of $S$ radii with region of validity of steady Stokes equations region (radius is $R_{s}$ ) and region of validity of quasi-steady Stokes equations (region radius is $\delta$ )

(Values for $\delta$ are close enough to $S_{w}$ for it to be said that they are of the same order, because the values of $S_{w}$ that would match $\delta$ can be obtained from velocity ratios within one standard deviation of the velocity ratios corresponding to the given values of $S_{w .}$.)

$\begin{array}{lccc}\text { Tetrahymena } & \text { P. caudatum } & \begin{array}{c}P . \text { multi- } \\ \text { micronucleatum }\end{array} \\ S_{o} & 108 a & 127 a & 132 a \\ R_{s} & 6100 a & 114 a & 70.5 a \\ S_{w} & 4.1 a & 0.64 a & 4.2 a \\ \delta & 2.25 a & 1 \cdot 10 a & 1.16 a \\ R_{s} / \delta & 2710 & 104 & 60.7 \\ S_{0} / S_{w} & 26.3 & 199 & 31.4\end{array}$

steady-motion Stokes equations. The results for swimming cells are much more encouraging, with relatively small difference between $S_{w}$ and $\delta$ for each ciliate. Accordingly, $S_{w}$ is proposed as a useful measure of $\delta$.

\section{SUMMARY}

It is generally assumed that wall drag on free-moving, self-propelled or passively moving micro-organisms is not significant under normal observation conditions. Yet the point at which such drag becomes significant has not been determined quantitatively.

By comparing the relative velocities of sinking as well as swimming ciliates in tubes of various bore widths it has been determined that wall drag on sinking cells is about $8 \%$ significant at 108-132 body radii (or minor semi-axes) from the cell surface while the corresponding range for swimming cells is less than $I-4 \cdot 2$ body radii.

These results are compared with the mathematical approximations for Stokes radius $R_{s}$ and depth of penetration of diffusing vorticity $\delta$ which characterize steady and quasi-steady Stokes flow respectively around a solid body. It is found that the asymptotic nature of the velocity profile of steady flow is reflected in the lack of agreement between $R_{s}$ and the measured distance for $8 \%$ drag. Conversely, the sharp gradient (or propulsive envelope) of the quasi-steady velocity profile is reflected in the substantial agreement between $\delta$ and the measured distance for $>0 \%$ drag.

It is suggested that the given formula for $\delta$ which includes allowance for a propagated wave is a valid measure of the thickness of the quasi-steady region and that observations on motile ciliates be restricted to organisms at least 4 cell radii from the nearest wall if measurements free of wall-drag effects are to be obtained.

\section{AP PENDIX}

\section{Meanings of Symbols}

a minor semi-axis of organism (body radius)

$c \quad$ half length of organism (major semi-axis)

$F_{s b} \quad$ Stokes drag on a body in a bounded fluid

$F_{s \infty} \quad$ Stokes drag on a body in an unbounded fluid

$R$ equivalent sphere radius 


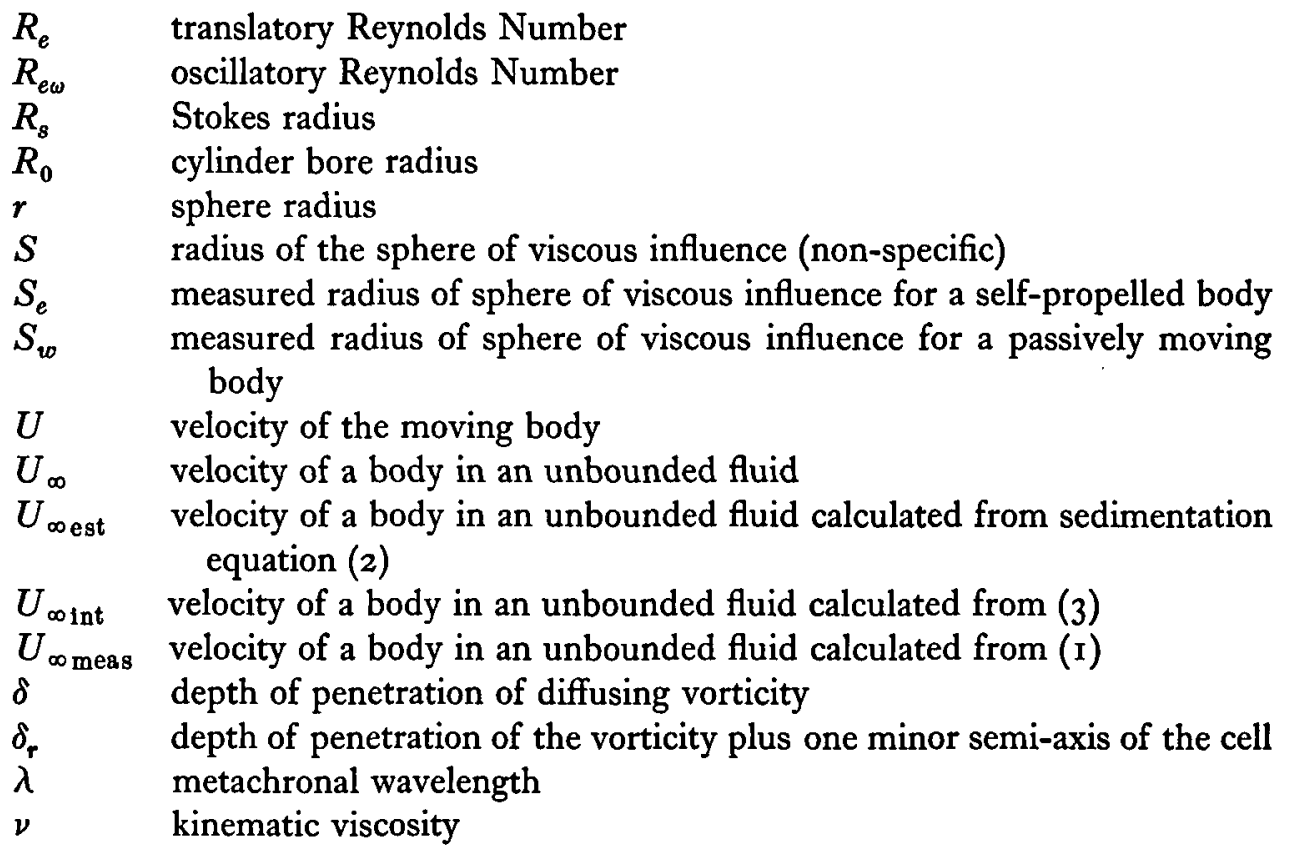

The author is indebted to Mr Long Wa Lee and Mr Stuart R. Keller for their assistance in performing the experiments. This investigation was supported by Grant $\mathrm{GK}_{31}$ I6IX from the National Science Foundation and Contract Noo014-67-A-00940012 from the Office of Naval Research.

\section{REFERENCES}

BlAKE, J. (1973). A finite model for ciliated microorganisms. $\mathcal{~}$. Biomechanics 6, 133-40.

Chwang, A., Winet, H. \& Wu, T. Y. (1973). A theoretical mechanism for Spirochetal locomotion. f. Mechanochem. Cell Motil. (in the press).

Chwang, A. T. \& Wu, T. Y. (197r). A note on the helical movement of microorganisms. Proc. Roy. Soc. Lond. B $178,327-46$.

Chwang, A., WU, T. Y. \& Winet, H. (1972). Locomotion of spirilla. Biophys. 7. 12, 1549-61.

Dunham, P. B. \& Child, F. M. (196I). Ion regulation in Tetrahymena. Biol. Bull. mar. biol. Lab., Woods Hole 121, 129-40.

Gray, J. (1962). Introduction: flagellar propulsion. In Spermatozoan Motility (ed. D. W. Bishop), pp. I-12. Washington: AAAS.

Gray, J. \& HaNCoCK, G. J. (1955). The propulsion of sea urchin spermatozoa. F. exp. Biol. 32, 802-14.

HAPPEL, J. \& Brenner, H. (1965). Low Reynolds Number Hydrodynamics. 534 pp. Englewood Cliffs: Prentice-Hall.

JAhn, T. L. \& VottA, J. J. (1972). Locomotion of Protozoa. Ann. Rev. Fluid Mech. 4, 93-1 I6.

KuZNICKI, L. (1968). Behavior of Paramecium in gravity fields. I. Sinking of immobilized specimens. Acta Protozool. 6, ro9-17.

MACHEMER, H. (1972). Ciliary activity and the origin of metachrony in Paramecium; effects of increased viscosity. 7. exp. Biol. 57, 239-6o.

Parducz, B. (1966). Ciliary movement and coordination in ciliates. Int. Rev. Cytol. 21, 91-128.

Preston, J. T. (I972). Determination of a Continuous Helical Ciliary Beat in Tetrahymena pyriformis and the Cytotic Effect of Serum Complement from Normal and Cystic Fibrotic Sera on the Organism. Doctoral Thesis, University of California, Los Angeles. $5^{8} \mathrm{pp}$.

Rosenhead, L. (ed.) (1963). Laminar Boundary Layers. Oxford University Press. 687 pp.

TAYLOR, G. I. (1952). The action of waving cylindrical tails in propelling microscopic organisms. Proc. Roy. Soc. Lond. A 211, 225-39.

WINET, H. (1969). The influence of gravity and origin of bioconvection in Tetrahymena pyriformis cultures. Doctoral Thesis, University of California, Los Angeles. 274 pp. 